$2008 / 04 / 07$

\title{
Yet another generalization of Postnikov's hook length formula for binary trees
}

\author{
Guo-Niu HAN
}

\begin{abstract}
We discover another one-parameter generalization of Postnikov's hook length formula for binary trees. The particularity of our formula is that the hook length $h_{v}$ appears as an exponent. As an application, we derive another simple hook length formula for binary trees when the underlying parameter takes the value $1 / 2$.
\end{abstract}

\section{Introduction}

Consider the set $\mathcal{B}(n)$ of all binary trees with $n$ vertices. For each vertex $v$ of $T \in \mathcal{B}(n)$ the hook length of $v$, denoted by $h_{v}$, or just $h$ for short, is the number of descendants of $v$ (including $v$ ). The following hook length formula for binary trees

$$
\sum_{T \in \mathcal{B}(n)} \prod_{v \in T}\left(1+\frac{1}{h_{v}}\right)=\frac{2^{n}}{n !}(n+1)^{n-1}
$$

was discovered by Postnikov [Po04]. Further combinatorial proofs and extensions have been proposed by several authors [CY08, GS06, MY07, Se08]. In particular, Lascoux conjectured the following one-parameter generalization

$$
\sum_{T \in \mathcal{B}(n)} \prod_{v \in T}\left(x+\frac{1}{h_{v}}\right)=\frac{1}{(n+1) !} \prod_{k=0}^{n-1}((n+1+k) x+n+1-k),
$$

which was subsequently proved by Du-Liu [DL08]. The latter generalization appears to be very natural, because the left-hand side of (2) can be obtained from the left-hand side of (1) by replacing 1 by $x$.

It is also natural to look for an extension of (1) by introducing a new variable $z$ in the right-hand side, namely by replacing $2^{n}(n+1)^{n-1} / n$ ! by $2^{n} z(n+z)^{n-1} / n$ ! It so happens that the corresponding left-hand side is also a sum on binary trees, but this time the hook length $h_{v}$ appears as an exponent. The purpose of this Note is to prove the following Theorem.

Theorem 1. For each positive integer $n$ we have

$$
\sum_{T \in \mathcal{B}(n)} \prod_{v \in T} \frac{(z+h)^{h-1}}{h(2 z+h-1)^{h-2}}=\frac{2^{n} z}{n !}(n+z)^{n-1} .
$$

With $z=1$ in (3) we recover Postnikov's identity (1). The following corollary is derived from our identity (3) by taking $z=1 / 2$. 
Corollary 2. For each positive integer $n$ we have

$$
\sum_{T \in \mathcal{B}(n)} \prod_{v \in T}\left(1+\frac{1}{2 h}\right)^{h-1}=\frac{(2 n+1)^{n-1}}{n !} .
$$

\section{Proof of the Theorem}

Let us take an example before proving the Theorem. There are five binary trees with $n=3$ vertices:
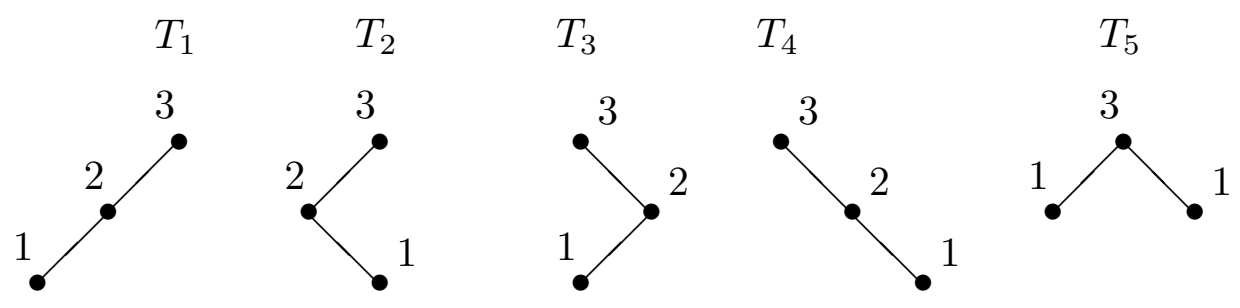

The hook lengths of $T_{1}, T_{2}, T_{3}, T_{4}$ are all the same $1,2,3$; but the hook lengths of $T_{5}$ are 1,1,3. The left-hand side of (3) is then equal to

$4 \times \frac{1}{(2 z)^{-1}} \cdot \frac{(z+2)^{1}}{2} \cdot \frac{(z+3)^{2}}{3(2 z+1)}+\frac{1}{(2 z)^{-1}} \cdot \frac{1}{(2 z)^{-1}} \cdot \frac{(z+3)^{2}}{3(2 z+1)}=\frac{2^{3} z(z+3)^{2}}{3 !}$.

Let $y(x)$ be a formal power series in $x$ such that

$$
y(x)=e^{x y(x)} .
$$

By the Lagrange inversion formula $y(x)^{z}$ has the following explicit expansion:

$$
y(x)^{z}=\sum_{n \geq 0} z(n+z)^{n-1} \frac{x^{n}}{n !} .
$$

Since $y^{2 z}=\left(y^{z}\right)^{2}$ we have

$$
\sum_{n \geq 0} 2 z(n+2 z)^{n-1} \frac{x^{n}}{n !}=\left(\sum_{n \geq 0} z(n+z)^{n-1} \frac{x^{n}}{n !}\right)^{2} .
$$

Comparing the coefficients of $x^{n}$ on both sides of (7) yields the following Lemma. 
Lemma 3. We have

$$
\frac{2 z(n+2 z)^{n-1}}{n !}=\sum_{k=0}^{n} \frac{z(k+z)^{k-1}}{k !} \times \frac{z(n-k+z)^{n-k-1}}{(n-k) !} .
$$

Proof of the Theorem. Let

$$
P(n)=\sum_{T \in \mathcal{B}(n)} \prod_{v \in T} \frac{(z+h)^{h-1}}{h(2 z+h-1)^{h-2}} .
$$

With each binary tree $T \in \mathcal{B}(n)(n \geq 1)$ we can associate a triplet $\left(T^{\prime}, T^{\prime \prime}, u\right)$, where $T^{\prime} \in \mathcal{B}(k)(0 \leq k \leq n-1), T^{\prime \prime} \in \mathcal{B}(n-1-k)$ and $u$ is a vertex of hook length $h_{u}=n$. Hence

$$
P(n)=\sum_{k=0}^{n-1} P(k) P(n-1-k) \times \frac{(z+n)^{n-1}}{n(2 z+n-1)^{n-2}} .
$$

It is routine to verify that $P(n)=2^{n} z(z+n)^{n-1} / n$ ! for $n=1,2,3$. Suppose that $P(k)=2^{k} z(z+k)^{k-1} / k$ ! for $k \leq n-1$. From identity (9) and Lemma 3 we have

$$
\begin{aligned}
P(n) & =\sum_{k=0}^{n-1} \frac{2^{k} z(z+k)^{k-1}}{k !} \times \frac{2^{n-k-1} z(z+n-k-1)^{n-k-2}}{(n-k-1) !} \\
& \times \frac{(z+n)^{n-1}}{n(2 z+n-1)^{n-2}} \\
= & \frac{2^{n} z}{n !}(z+n)^{n-1} .
\end{aligned}
$$

By induction, formula (3) is true for any positive integer $n$.

\section{Concluding and Remarks}

The right-hand sides of (3) and (4) have been studied by other authors [GS06, DL08, MY07], but our formula has the following two major differences: (i) the hook length $h_{v}$ appears as an exponent; (ii) the underlying set remains the set of binary trees, whereas in the above mentioned papers the summation has been changed to the set of $m$-ary trees or plane forests. It is interesting to compare Corollary 2 with the following results obtained by Du and Liu [DL08]. Note that the right-hand sides of formulas (4), (10) and (11) are all identical! 
Proposition 4. For each positive integer $n$ we have

$$
\sum_{T \in \mathcal{T}(n)} \prod_{v \in I(T)}\left(\frac{2}{3}+\frac{1}{3 h}\right)=\frac{(2 n+1)^{n-1}}{n !}
$$

where $\mathcal{T}(n)$ is the set of all 3-ary trees with $n$ internal vertices and $I(T)$ is the set of all internal vertices of $T$.

Proposition 5. For each positive integer $n$ we have

$$
\sum_{T \in \mathcal{F}(n)} \prod_{v \in(T)}\left(2-\frac{1}{h}\right)=\frac{(2 n+1)^{n-1}}{n !}
$$

where $\mathcal{F}(n)$ is the set of all plane forests with $n$ vertices.

\section{References}

[CY08] Chen, William Y.C.; Yang, Laura L.M., On Postnikov's hook length formula for binary trees, European Journal of Combinatorics, in press, 2008.

[DL08] Du, Rosena R. X.; Liu, Fu, $(k, m)$-Catalan Numbers and Hook Length Polynomials for Plane Trees, European J. Combin, 28 (2007), pp. 1312-1321.

[GS06] Gessel, Ira M.; Seo, Seunghyun, A refinement of Cayley's formula for trees, arXiv:math. $\mathrm{CO} / 0507497$

[MY07] Moon, J. W.; Yang, Laura L. M., Postnikov identities and Seo's formulas, Bull. Inst. Combin. Appl., 49 (2007), pp. 21-31.

[Po04] Postnikov, Alexander, Permutohedra, associahedra, and beyond, arXiv:math. CO/0507163, 2004.

[Se08] Seo, Seunghyun, A combinatorial proof of Postnikov's identity and a generalized enumeration of labeled trees, arXiv:math.CO/0409323.

I.R.M.A. UMR 7501

Université Louis Pasteur et CNRS,

7, rue René-Descartes

F-67084 Strasbourg, France

guoniu@math.u-strasbg.fr 